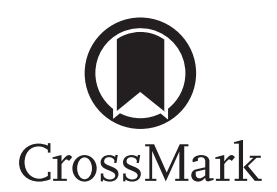

\title{
Do admission glucose levels aid in predicting mortality associated with community-acquired pneumonia?
}

\author{
To the Editor:
}

Diabetes mellitus increases the risk of and mortality associated with community-acquired pneumonia (CAP) [1]. Hyperglycaemia negatively impacts both the innate and adaptive immune response, which could lead to decreased bacterial clearance and increased mortality from infection [2]. This can be extended to non-diabetic patients as well: hyperglycaemia on admission is an independent risk factor for higher complication rates, longer length of stay, and higher mortality in diabetics and non-diabetics with CAP [3-6]. Hypoglycaemia on admission has also been associated with an independent increased risk of mortality in patients with CAP [7, 8]. Currently, two severity scoring systems are widely used for predicting CAP mortality: the pneumonia severity index (PSI) and CURB-65 score (confusion, urea $>7 \mathrm{mmol} \cdot \mathrm{L}^{-1}$, respiratory rate $\geqslant 30$ breaths $\cdot \mathrm{min}^{-1}$, blood pressure $<90 \mathrm{mmHg}$ (systolic) or $\leqslant 60 \mathrm{mmHg}$ (diastolic), age $\geqslant 65$ years) [5,9]. Admission glucose levels (AGL) are part of the PSI, as a categorical variable for hyperglycaemia, but not of CURB-65. We, therefore, investigated whether AGL contributes to these severity scores when both hypo- and hyperglycaemic states are appropriately modelled.

This study was conducted with data from the CAP-START trial, a multicentre cluster-randomised cross-over trial designed to evaluate three empiric antibiotic strategies for patients with CAP admitted to non-ICU wards [10].

The study population consisted of patients aged 18 years or older, admitted with a clinical diagnosis of CAP to non-ICU wards, with evidence of a new pulmonary infiltrate on chest radiography or computed tomography (CT). Patients or relatives provided written informed consent for review of their medical charts. Standardised case record forms were used to extract information such as patient demographics, comorbidities and clinical characteristics from medical records. Trained research nurses acquired all data. Routinely measured non-fasting AGL on initial presentation were recorded when available. Multiple imputation with 10 imputed datasets was performed for variables missing (completely) at random.

We used logistic regression to assess associations with 30-day mortality. AGL was truncated for the 1st and 99th percentile to minimise the effect of outliers. PSI and CURB-65 scores were calculated for each individual patient; we calculated an adjusted version of the PSI, in which the dichotomised criterion for AGL $>14 \mathrm{mmol} \cdot \mathrm{L}^{-1}$ was not taken into account, to optimally assess the added value of AGL as a continuous variable. We determined the appropriate modelling form for AGL in two steps. First, we visually checked the association between AGL and 30-day mortality by plotting locally weighted regression (LOESS) curves. As we expected a non-linear effect on our outcome, we used fractional polynomials to estimate the most appropriate functional form. We applied the technique as proposed by MorRIs et al. to combine estimation and selection of the fractional polynomial fit with multiple imputation [11].

To determine the performance of our models we calculated the area-under-the-receiver-operating-curve (AUC) and Brier scores. We created confidence intervals by bootstrapping with 2000 samples. Differences in AUCs between nested models were tested using the approach of RoBIN et al. [12]. All analyses were performed in $\mathrm{R}$ software for statistical computing, version 3.2.0, using the libraries MICE, $p R O C$ and $m f p$. $\mathrm{P}$-values $<0.05$ were considered statistically significant.

@ERSpublications

Admission glucose levels do not improve discrimination of current severity scores in communityacquired pneumonia http://ow.ly/Dyrz30f7Bwi

Cite this article as: Postma DF, van Werkhoven CH, Troeman DPR, et al. Do admission glucose levels aid in predicting mortality associated with community-acquired pneumonia? Eur Respir J 2017; 50: 1700307 [https://doi.org/10.1183/13993003.00307-2017]. 
Of 2283 patients included in the CAP-START study, 1737 had radiologically confirmed CAP. After exclusion of a single hospital in which glucose levels were missing for 53\% cases, 1549 patients remained for analysis.

996 patients $(57.3 \%)$ were male, median age was 70 years (IQR 58-79), and $82.9 \%$ had one or more comorbidities, particularly chronic pulmonary disease (45.2\%), chronic cardiovascular disease (22\%) and cancer (16.7\%). The median AGL was $7.1 \mathrm{mmol} \cdot \mathrm{L}^{-1}$ (IQR 6.2-8.5). The median CURB-65 score was 1 (IQR 1-2) and mean PSI score was 88 (SD 31.3). The median length of stay in hospital was 6 days (IQR 4-9). The 30-day mortality rate was $5.9 \%(n=92)$. When stratifying for admission glucose categories, increasing proportions of prior diabetes, insulin use, and higher PSI and CURB-65 scores were noted in the hypo- and hyperglycaemic states on admission. Patients with hypo- or hyperglycaemia had higher 30-day mortality rates than patients with normoglycaemia (table 1). Fractional polynomial fits of AGLs on 30-day mortality were superior to linear functions. The predicted risk on mortality rises with increasing (glucose levels $>7 \mathrm{mmol} \cdot \mathrm{L}^{-1}$ ) as well as decreasing AGLs (glucose levels $<7 \mathrm{mmol} \cdot \mathrm{L}^{-1}$ ). To describe estimated risks from the models, we calculated odds ratios using $7 \mathrm{mmol} \cdot \mathrm{L}^{-1}$ as a reference value, and fitting centred values of other categories (table 1). AGL significantly increased the fit of models with the PSI and CURB-65 score, with p-values of 0.001 and $<0.001$, respectively. After addition of AGL to the PSI and CURB-65 model, their AUCs increased from 0.747 (95\% CI: $0.695-0.792)$ to 0.764 (95\% CI: $0.714-0.811)$ and from $0.697(0.647-0.747)$ to $0.722(0.666-0.772)$, respectively. Yet, these models were not significantly different from the models without AGL. The Brier scores were also not different between the models with or without AGL for both PSI, 0.053 (95\% CI: 0.044-0.063) versus 0.052 (95\% CI: 0.044-0.061) and CURB-65, 0.055 (95\% CI: 0.045-0.065) versus 0.054 (95\% CI: 0.044-0.062).

In this cohort of 1549 patients admitted to non-ICU wards with radiologically confirmed CAP, AGL predicted 30-day mortality independently from PSI or CURB-65 scores, but when added to these widely used scores, AGL hardly improved discriminative power.

The association between hypoglycaemia and death has been reported before in CAP patients [7, 8]. Diabetes or insulin treatment may underlie part of this association, yet hypoglycaemia may also occur during sepsis, in which impaired hepatic gluconeogenesis, increasing peripheral glucose utilisation, or relative adrenal insufficiency might explain the association with adverse outcomes in non-diabetic patients [13].

Our results are concordant with previous prognostic research in which hyperglycaemia on admission has been associated with increased mortality $[3,4,6]$. Admission hyperglycaemia might reflect chronic hyperglycaemia in pre-existing or undiagnosed diabetes mellitus, which increases risks of adverse outcome [14]. Naturally, other pathophysiologic mechanisms could also contribute to the observed association. Hyperglycaemia might reflect disease severity, as a stronger inflammatory response leads to more insulin resistance, and consequently higher serum glucose levels [15]. Still, high glucose levels have also been linked to direct toxic effects on components of the immune system, e.g. chemotaxis, phagocytosis, macrophage activation, and complement functions, which might impair resolution of the infection and subsequently prognosis [2].

Although AGLs improved the model fit of the PSI and CURB-65 model, the increase in AUC was non-significant, and the marginal changes in Brier scores indicate that the actual predicted probabilities of

TABLE 1 Model-based estimates for 30-day mortality in reference to categories of admission glucose levels

\begin{tabular}{|c|c|c|c|c|c|c|}
\hline $\begin{array}{l}\text { Non-fasting } \\
\text { AGL mmol. } \mathrm{L}^{-1}\end{array}$ & \multicolumn{2}{|c|}{ Patients } & Ref. value & $\begin{array}{l}\text { Bivariate analysis } \\
\text { (Model A) }\end{array}$ & $\begin{array}{l}\text { Adjusted for PSI } \\
\text { score (Model B) }\end{array}$ & $\begin{array}{l}\text { Adjusted for } \\
\text { CURB-65 score } \\
\text { (Model C) }\end{array}$ \\
\hline $6-<8$ & 733 & $38(5.2)$ & 7 & Ref. & & \\
\hline $8-<10$ & 277 & $10(3.6)$ & 9 & $1.29(1.09-1.54)$ & $1.08(0.91-1.27)$ & $1.10(0.94-1.29)$ \\
\hline $10-<12$ & 98 & $8(8.2)$ & 11 & $1.84(1.33-2.54)$ & $1.33(0.97-1.81)$ & $1.40(1.04-1.90)$ \\
\hline
\end{tabular}

Data in the first two columns are from a single imputation dataset. Model-based estimates of ORs (95\% Cls) for 30-day mortality for several admission glucose level (AGL) categories. ORs were calculated for reference (Ref.) values in the centre of categories. Model A is for a second-degree polynomial fit of AGL only. Models B and C are the adjusted models with pneumonia severity index (PSI) and CURB-65 scores (confusion, urea $>7 \mathrm{mmol} \cdot \mathrm{L}^{-1}$, respiratory rate $\geqslant 30$ breaths $\cdot \mathrm{min}^{-1}$, blood pressure $<90 \mathrm{mmHg}$ (systolic) or $\leqslant 60 \mathrm{mmHg}$ (diastolic), age $\geqslant 65$ years), respectively. Formulas for the final logistic regression models, in which $\mathrm{x}$ denotes admission glucose level, $\beta$ denotes model betas for CURB-65 categories, and $z$ denotes PSI score, were as follows. Model $A: \ln O R=-0.64-2.07 \cdot(x / 10)^{-2}-2.36 \cdot(x / 10)^{-2} \cdot \log (x / 10)$. Model $B$ : $\ln \mathrm{OR}=-3.15+0.03 \cdot \mathrm{z}+1.58 \cdot(\mathrm{x} / 10)^{-2}-4.23 \cdot(\mathrm{x} / 10)^{-1}$. Model $\mathrm{C}: \ln \mathrm{OR}=-1.23+\beta+1.77 \cdot(\mathrm{x} / 10)^{-2}-4.79 \cdot(\mathrm{x} / 10)^{-1}$. Model C betas were $0.92(\mathrm{CURB}$ 65 score 1), 1.63 (CURB 65 score 2), 2.42 (CURB 65 score 3 ), 1.88 (CURB 65 score 4 ) and 3.17 (CURB 65 score 5 ). 
the models with and without AGLs are virtually identical. Thus, these absolute differences had little clinical relevance. Still, future research on prognostic modelling in CAP should preferably evaluate the non-linear association of AGL with outcomes.

Several study limitations have to be considered. First, our analyses were limited to patients admitted to non-ICU wards. However, as this concerns the majority of hospitalised CAP and some physicians use these scores to guide treatment decisions, our findings remain relevant. Second, only a small number of patients had AGLs $<4 \mathrm{mmol} \cdot \mathrm{L}^{-1}$; this may have hampered estimation of the best fitting model, explaining the higher predicted OR for $9 \mathrm{mmol} \cdot \mathrm{L}^{-1}$ (8-10 category), while the observed mortality was actually lower in this category.

In conclusion, both hyper- and hypoglycaemia at the time of admission increase the risk of 30-day mortality in CAP patients admitted to non-ICU wards. A model of this U-shaped association performs significantly better than a linear association. Yet, the addition of this U-shaped association for AGLs to both PSI- or CURB-65 scores did not increase the predictive capacity of these scores in our population.

Douwe F. Postma ${ }^{1,2,3}$, Cornelis H. van Werkhoven $^{1}$, Darren P.R. Troeman ${ }^{1}$, Sanjay U.C. Sankatsing ${ }^{3}$, Jan Jelrik Oosterheert ${ }^{2}$ and Marc J.M. Bonten ${ }^{1,4}$

${ }^{1}$ Julius Centre for Health Sciences and Primary Care, University Medical Centre Utrecht, Utrecht University, Utrecht, The Netherlands. ${ }^{2}$ Dept of Internal Medicine and Infectious Diseases, University Medical Centre Utrecht, Utrecht University, Utrecht, The Netherlands. ${ }^{3}$ Dept of Internal Medicine, Diakonessenhuis Utrecht, Utrecht, The Netherlands. ${ }^{4}$ Dept of Medical Microbiology, University Medical Centre Utrecht, Utrecht University, Utrecht, The Netherlands.

Correspondence: Douwe F. Postma, Dept of Internal Medicine and Infectious Diseases, University Medical Centre Utrecht, PO BOX 85500/3508 GA Utrecht, The Netherlands. E-mail: d.f.postma@umcutrecht.nl

Received: May 192016 | Accepted after revision: July 212017

Support statement: Supported by a grant (171202002) from the Netherlands Organization for Health Research and Development. Funding information for this article has been deposited with the Crossref Funder Registry.

Conflict of interest: Disclosures can be found alongside this article at erj.ersjournals.com

Acknowledgements: We would like to thank the members of the CAP-START study group for securing the conduct of the study in all participating hospitals.

Additional thanks to Tim P. Morris, MRC Clinical Trials Unit at University College London, for his guidance on applying fractional polynomial estimation and selection in a multiple imputation context.

\section{References}

1 Shah BR, Hux JE. Quantifying the risk of infectious diseases for people with diabetes. Diabetes Care 2003; 26 : 510-513.

2 Schuetz P, Castro P, Shapiro NI. Diabetes and sepsis: preclinical findings and clinical relevance. Diabetes Care 2011; 34: 771-778.

3 Lepper PM, Ott S, Nüesch E, et al. Serum glucose levels for predicting death in patients admitted to hospital for community acquired pneumonia: prospective cohort study. BMJ 2012; 344: e3397.

4 Schuetz P, Friedli N, Grolimund E, et al. Effect of hyperglycaemia on inflammatory and stress responses and clinical outcome of pneumonia in non-critical-care inpatients: results from an observational cohort study. Diabetologia 2014; 57: 275-284.

5 Fine MJ, Auble TE, Yealy DM, et al. A prediction rule to identify low-risk patients with community-acquired pneumonia. N Engl J Med 1997; 336: 243-250.

6 McAlister FA, Majumdar SR, Blitz S, et al. The relation between hyperglycemia and outcomes in 2,471 patients admitted to the hospital with community-acquired pneumonia. Diabetes Care 2005; 28: 810-815.

7 Gamble J-M, Eurich DT, Marrie TJ, et al. Admission hypoglycemia and increased mortality in patients hospitalized with pneumonia. Am J Med 2010; 123: 556e.11-e16.

8 Singanayagam A, Chalmers JD, Hill AT. Admission hypoglycaemia is associated with adverse outcome in community-acquired pneumonia. Eur Respir J 2009; 34: 932-939.

9 Lim WS, van der Eerden MM, Laing R, et al. Defining community acquired pneumonia severity on presentation to hospital: an international derivation and validation study. Thorax 2003; 58: 377-382.

10 Postma DF, van Werkhoven $\mathrm{CH}$, van Elden LJR, et al. Antibiotic treatment strategies for community-acquired pneumonia in adults. N Engl J Med 2015 ; 372: 1312-1323.

11 Morris TP, White IR, Carpenter JR, et al. Combining fractional polynomial model building with multiple imputation. Stat Med 2015; 34: 3298-3317.

12 Robin X, Turck N, Hainard A, et al. pROC: an open-source package for R and S+ to analyze and compare ROC curves. BMC Bioinformatics 2011; 12: 77.

13 McGeough EC, Walker JD. Hypoglycaemia with septicaemia: 2 case reports and a review of the literature. Scott Med J 2006; 51: 57-57.

14 MacIntyre EJ, Majumdar SR, Gamble J-M, et al. Stress hyperglycemia and newly diagnosed diabetes in 2124 patients hospitalized with pneumonia. Am J Med 2012; 125: e17-e23.

15 Dungan KM, Braithwaite SS, Preiser J-C. Stress hyperglycaemia. Lancet 2009; 373: 1798-1807. 